\title{
Generic sets in definably compact groups
}

\author{
by \\ Ya'acov Peterzil (Haifa) and \\ Anand Pillay (Urbana, IL, and Leeds)
}

\begin{abstract}
A subset $X$ of a group $G$ is called left generic if finitely many left translates of $X$ cover $G$. Our main result is that if $G$ is a definably compact group in an o-minimal structure and a definable $X \subseteq G$ is not right generic then its complement is left generic.

Among our additional results are (i) a new condition equivalent to definable compactness, (ii) the existence of a finitely additive invariant measure on definable sets in a definably compact group $G$ in the case where $G={ }^{*} H$ for some compact Lie group $H$ (generalizing results from [1]), and (iii) in a definably compact group every definable subsemi-group is a subgroup.

Our main result uses recent work of Alf Dolich on forking in o-minimal stuctures.
\end{abstract}

1. Introduction. In his paper [9], the second author formulated a conjecture on definably compact groups in (saturated) o-minimal structures. The conjecture says, roughly, that such a group $G$ has a smallest typedefinable subgroup $G^{00}$ of bounded index, and that $G / G^{00}$, when equipped with the "logic topology", is a compact Lie group whose dimension equals the o-minimal dimension of $G$. In [2] the existence of the smallest typedefinable subgroup of bounded index $G^{00}$ was established, as well as the fact that $G / G^{00}$ is a compact Lie group. However, it remains open whether $G^{00}$ is at all different from $G$ and, in particular, if the Lie group $G / G^{00}$ and $G$ have the same dimension. When restricted to commutative $G$, the second (as yet unproved) part of the conjecture is equivalent to " $G$ " is torsion-free".

One of the missing ingredients towards establishing the full conjecture seems to be a good understanding of "generic" definable sets in definably

2000 Mathematics Subject Classification: 03C64, 22E15.

Key words and phrases: o-minimal structure, definably compact group, generic set, Haar measure, forking.

The first author was partially supported by funds from NSF Focused Research Grant DMS 0100979. The second author was partially supported by NSF grants DMS 0300639 and DMS 0100979. 
compact groups, where the definable set $X \subseteq G$ is said to be generic if finitely many group translates of $X$ by elements of $G$ cover $G$. In the context of $\omega$-stable groups $X \subseteq G$ is generic if and only if it has the same Morley rank as $G$. The obvious analogue, in the case of groups in o-minimal structures, immediately fails as every bounded open interval in $\langle\mathbb{R},+\rangle$ has full dimension but it is clearly not generic. It might seem at first that the only obstacle is the fact the group is not definably compact, as in compact Lie groups every open set is generic. However, even if we assume that $G$ is a definably compact group it is not true that subsets of $G$ of full dimension are generic, once we leave the context of the real numbers. Indeed, if we take $G$ to be $S^{1}$, in a nonarchimedean extension of $\langle\mathbb{R},<,+, \cdot\rangle$, then an "infinitesimal interval" in $G$ has dimension 1 but is not generic.

In this note we present some new basic properties of generic sets in definably compact groups. We concentrate on the case of commutative groups and show that the union of two nongeneric sets in a definably compact group is nongeneric. Our results rely on the work of Dolich (see [4]) on forking in o-minimal structures (we include in an appendix a complete proof of the needed results). We then present some corollaries regarding definable semigroups in definably compact groups, and a conjecture on generic sets which, as we show, implies the full group conjecture mentioned above.

We also study the case where our saturated o-minimal structure $\mathcal{M}$ is an expansion of an elementary extension of an o-minimal structure $\mathcal{M}_{0}=$ $\langle\mathbb{R},<,+, \cdot, \ldots\rangle$, and the definably compact group $G$ is the interpretation in $\mathcal{M}$ of a formula defining a compact Lie group $G(\mathbb{R})$ in $\mathcal{M}_{0}$. We relate genericity of definable (in $\mathcal{M}$ ) subsets $X$ of $G$ to genericity of $\operatorname{st}(X)$ (the image of $X$ under the standard part map) in $G(\mathbb{R})$ and develop a "Haar measure" for definable subsets of $G$.

Acknowledgements. The first author would like to thank the Department of Mathematics and the logic group at the University of Illinois at Urbana-Champaign for their hospitality during the 2003-2004 academic year.

The second author would like to thank Alessandro Berarducci, Margarita Otero, and Ludomir Newelski for various discussions and communications which influenced and motivated some of the work presented in this paper.

1.1. Assumptions. We work in a saturated o-minimal structure $\mathcal{M}$ expanding a real closed field. Let us say a few words about this assumption.

As is shown in [5], every definable regular space in an o-minimal expansion of a real closed field (see Chapter 10 in [5] for definitions) is definably homeomorphic to a subset of $M^{n}$ for some $n$. Such a space is called affine. It was pointed out in [6] that every definable manifold which is Hausdorff is necessarily regular, and therefore every definable group in $\mathcal{M}$, equipped 
with its group topology (see [8]), is affine. Namely, we may assume that the underlying set of the group is a definable subset of $M^{n}$, for some $n$, and the topology induced from $M^{n}$ makes the group into a topological group.

This is our only use of the assumption that $\mathcal{M}$ is an expansion of a real closed field. Moreover, even though the proofs (mainly of Dolich's results) make use of the fact that the group is affine, most of the statements themselves which we prove can be formulated for any definable group. Thus, the main results (such as Corollary 3.9) will still be true under the weaker assumption that $\operatorname{Th}(\mathcal{M})$ can be expanded to an o-minimal expansion of a real closed field. For example, if $\mathcal{M}$ is an ordered vector space over an ordered field.

2. Dolich's work and some corollaries. Our results depend heavily on Alfred Dolich's work [4], where he analyzes the notions of dividing and forking in o-minimal structures. We are going to use the following theorem, which is a straightforward corollary of his work (the results in his paper are substantially stronger).

Theorem 2.1. Suppose $X$ is a definable closed and bounded subset of $M^{n}$, and $\mathcal{M}_{0}$ is a model (small elementary substructure of $\mathcal{M}$ ). Then the following are equivalent:

(1) The set of $\mathcal{M}_{0}$-conjugates of $X$ is finitely consistent.

(2) $X$ has a point in $\mathcal{M}_{0}$.

Since Dolich's paper does not contain the precise formulation of the above theorem, we include in an appendix to our paper a direct proof (which is extracted from Dolich's paper). Moreover, even though Dolich's original assumptions are that $M$ expands a real closed field (or some equivalent assumption), it turns out that the proof of the theorem does not require this assumption. It actually follows from Dolich's work that for a closed and bounded set $X$, the above two properties are equivalent to $X$ not forking over $\mathcal{M}_{0}$, but we will not make use of this fact here.

The following results on definable families of sets follow from Theorem 2.1 by compactness and by taking complements.

Corollary 2.2.

(i) Assume that $\mathcal{F}=\left\{F_{s}: s \in S\right\}$ is a definable family of closed and bounded sets ( $S$ is now a definable set), with the finite intersection property. If $\mathcal{F}$ is definable over $\mathcal{M}_{0}$ then there are finitely many elements $a_{1}, \ldots, a_{k} \in \mathcal{M}_{0}$ such that every $F_{s}$ contains one of the $a_{i}$ 's.

(ii) Assume that $\mathcal{U}=\left\{U_{s}: s \in S\right\}$ is a definable family of open subsets of a closed and bounded set $X$, with the property that every finite subset of $X$ is contained in one of the $U_{s}$ 's. Then $\mathcal{U}$ has a finite subcover. 
2.1. Some corollaries for definably compact sets. In [7] the notion of a definably compact space was introduced. A space $X$ is called definably compact if every definable map $\gamma:(0,1) \rightarrow X$ has a limit in $X$ as $\gamma$ tends to 0 . It was shown there that if $X$ is affine then it is definably compact if and only if it is closed and bounded. Using Dolich's work we can now state an equivalent definition, in the language of covers.

For a definable set $X \subseteq M^{n}$, we say that a family $\left\{U_{s}: s \in P\right\}$ of definable open subsets of $X$ is a definable open cover of $X$ parameterized by a complete type over $A$ if the family is uniformly definable, covers $X$, and $P$ is the set of realizations of a complete $m$-type over $A$. Said differently, there is a formula $\phi(x, y)$, with length $x=n$ and length $y=m$, and there is a complete type $p \in S^{m}(A)$, such that

(i) for every $a \in p(M), \phi(x, a)$ defines an open subset of $X$,

(ii) for every element $x \in X$ there is $a \in P$ such that $\phi(x, a)$ holds.

Corollary 2.3. Let $X \subseteq M^{n}$ be a definable set, definable over a model $\mathcal{M}_{0}$ say. Then the following are equivalent:

(1) $X$ is definably compact.

(2) If $\left\{U_{s}: s \in P\right\}$ is a definable open cover of $X$ parameterized by a complete type over $\mathcal{M}_{0}$, then it contains a finite subcover of $X$.

Proof. $(1) \Rightarrow(2)$ is immediate from Theorem 2.1, by taking complements.

For the opposite direction, assume that $X$ is not definably compact, and let $\gamma:(0,1) \rightarrow X$ be a definable continuous map such that $\lim _{t \rightarrow 1} \gamma(t)$ does not exist in $X$ (we can always assume that $\gamma$ is continuous, by o-minimality). In particular, $\gamma$ is not a constant function. It follows that for every $s \in(0,1)$, the set $F_{s}=\gamma([s, 1))$ is closed in $X$ and we have $F_{s} \subsetneq F_{t}$ whenever $t<s$.

Let $p(y)$ be the complete type of $s$ over $\mathcal{M}_{0}$ where $s<1$ and $s>$ $\mathcal{M}_{0} \cap(-\infty, 1)$. Then the family $\left\{U_{s}=X \backslash F_{s}: s \in P\right\}$ is a definable open cover of $X$ parameterized by a complete type over $\mathcal{M}_{0}$. It is easy to see that it has no finite subcover.

EXAMPLE. Let $\mathcal{M}_{0}$ be a nonarchimedean elementary extension of $\langle\mathbb{R},+, \cdot\rangle$ and let $\alpha>0$ be an infinitesimal element of $\mathcal{M}_{0}$. For $a, b \in[0,1]$, let $X(a, b)=[0,1] \backslash((a-\alpha, a+\alpha) \cup(b-\alpha, b+\alpha))$. The family $\{X(a, b)$ : $a, b \in[0,1]\}$ has the finite intersection property. If we take $a_{1}, a_{2}, a_{3}$ to be any elements of $[0,1] \cap \mathbb{R}$ then every $X(a, b)$ must contain one of those. Notice that we need at least three such elements since for any two elements of $[0,1]$ there is $X(a, b)$ which does not contain any of those.

Applying compactness again to Corollary 2.3, we obtain the following corollary. It is not clear to us, even if we work in the structure $\langle\mathbb{R},+, \cdot\rangle$, how to get a direct proof of this result, without using Dolich's work. 
Corollary 2.4. Assume that we are given a definable family of families of definably compact sets. Namely, the following collection of sets is uniformly definable:

$$
\left\{\mathcal{F}_{t}=\left\{F_{s, t}: s \in S_{t}\right\}: t \in T\right\}
$$

where each $F_{s, t}$ is a closed and bounded set.

Then there are $L$ and $K$ such that for every $t \in T$, if the family $\mathcal{F}_{t}$ is $L$-consistent then there are $K$ elements in $\mathrm{dcl}(t)$ such that every set in $\mathcal{F}_{t}$ contains one of these elements.

Proof. We consider the partial type $p(s, t)$ determined by: $t \in T, s \in S_{t}$, " $\mathcal{F}_{t}$ has the finite intersection property" and

$$
\left\{f(t) \notin F_{s, t}: f \text { is } 0 \text {-definable }\right\} .
$$

By Theorem 2.1, the type $p(s, t)$ is inconsistent, from which the corollary follows.

3. Properties of generic sets. Recall the conventions from Section 1. In particular we work in a saturated o-minimal structure $\mathcal{M} ; \mathcal{M}_{0}$ will denote a small elementary substructure.

Throughout this section we assume that $G$ is a subset of $M^{n}$, whose group topology is induced from $M^{n}$. We also assume $G$ to be definably connected.

Note that although the proofs of most of the results will require this embedding assumption, the main statements proven do not involve the topology of $G$ at all, and thus will hold for any definable $G$ which, in some expansion, can be embedded into $M^{n}$.

We will often specialize to the case where $G$ is also definably compact, in which case we assume $G$ is a closed bounded subset of $M^{n}$.

Definition 3.1. $X \subseteq G$ is left (right) generic if finitely many left (right) translates of $X$ cover $G$. When $G$ is commutative we will just say generic.

We will use the following fact from [8]:

FACT 3.2. If $X$ is definable and $\operatorname{dim}(G \backslash X)<\operatorname{dim}(G)$ then $X$ is both left and right generic.

Remark 3.3. Suppose $G=(M,+)$. Then a definable $X \subseteq M$ is generic if and only if it is coinitial and cofinal in $M$. So there exist two nongeneric definable subsets of $G$ whose union is $G$.

LEMMA 3.4.

(i) If $X$ is (left or right) generic in $G$ then $\operatorname{dim}(X)=\operatorname{dim}(G)$.

(ii) $X$ is left (right) generic in $G$ if and only if $\mathrm{Cl}_{G}(X)$ is left (right) generic in $G\left(\right.$ by $\mathrm{Cl}_{G}(X)$ we mean the closure of $X$ in $\left.G\right)$. 
Proof. (i) Suppose $\operatorname{dim}(X)<\operatorname{dim}(G)$. Then $\operatorname{dim}(g X)<\operatorname{dim}(G)$ for all $g \in G$. Hence finitely many translates of $X$ could not cover $G$.

(ii) Suppose $\mathrm{Cl}_{G}(X)$ is generic. Let $Y$ be the frontier of $X$, that is, $\mathrm{Cl}_{G}(X) \backslash X$. Let $G=g_{1} \mathrm{Cl}_{G}(X) \cup \cdots \cup g_{m} \mathrm{Cl}_{G}(X)$. Then $Z=g_{1} Y \cup \cdots \cup g_{m} Y$ has dimension $<\operatorname{dim}(G)$. So by Fact 3.2, finitely many translates of $G \backslash Z$ cover $G$. So clearly finitely many translates of $X$ cover $G$.

Lemma 3.5. Assume that $G$ is an $\mathcal{M}_{0}$-definable group and $X$ an $\mathcal{M}_{0^{-}}$ definable subset of $G$. Then $X$ is left generic in $G$ if and only if every right translate of $X$ contains a point in $G\left(\mathcal{M}_{0}\right)$.

Proof. Assume that $X$ is left generic. Then there are $g_{1}, \ldots, g_{k} \in G\left(\mathcal{M}_{0}\right)$ such that $G=\bigcup_{i=1}^{k} g_{i} X$. It is immediate that every right translate of $X$ contains one of the $g_{i}^{-1}$ 's.

Assume now that $X$ is not left generic. Then for every $h_{1}, \ldots, h_{k} \in G$ there is $g \in G$ such that $h_{i} \notin X g$ for $i=1, \ldots, k$. By compactness, there is $g \in G$ such that $X g$ has no point in $G\left(\mathcal{M}_{0}\right)$.

The main ingredient of our work here is the following corollary to Theorem 2.1 .

Lemma 3.6. Assume that $G$ is a definable group, $X$ a definably compact subset of $G$, both defined over $\mathcal{M}_{0}$. Assume that, for some $g \in G, X g$ does not contain any element from $G\left(\mathcal{M}_{0}\right)$. Then there are $g_{1}, \ldots, g_{k}$, all realizing the type of $g$ over $\mathcal{M}_{0}$, such that $X g_{1} \cap \cdots \cap X g_{k}=\emptyset$. In particular, $G \backslash X$ is right generic.

Proof. Let $p=\operatorname{tp}\left(g / \mathcal{M}_{0}\right)$, and apply Theorem 2.1 to the family $\left\{X g^{\prime}\right.$ : $\left.g^{\prime} \models p\right\}$.

Theorem 3.7. Assume that $G$ is a definable group, and $X \subseteq G$ a definable set whose closure in $G$ is definably compact. If $X$ is not left generic then $G \backslash X$ is right generic.

Proof. By Lemma 3.4(ii), we may assume $X$ to be closed in $G$ and hence definably compact. Suppose $X$ and $G$ are defined over $\mathcal{M}_{0}$. Now Lemmas 3.5 and 3.6 imply the desired result.

Corollary 3.8. If $G$ is not definably compact, $X \subseteq G$ is definable, and $\mathrm{Cl}_{G}(X)$ is definably compact then $G \backslash X$ is both right and left generic.

Proof. Since $G$ is not definably compact, $\mathrm{Cl}_{G}(X)$ cannot be generic in $G$ (since a finite union of definably compact sets is definably compact as well), and hence $X$ itself can be neither left or right generic in $G$. By the last theorem $G \backslash \mathrm{Cl}_{G}(X)$ is generic, and hence $G \backslash X$ is.

Corollary 3.9. If $G$ is definably compact and commutative then the union of two nongeneric definable subsets of $G$ is also nongeneric. 
Proof. Suppose $X_{1}, X_{2}$ are definable subsets of $G$, and that $X=X_{1} \cup X_{2}$ is generic. So $\bigcup_{i=1}^{m} g_{i} X=G$ for some $g_{1}, \ldots, g_{m} \in G$. But $\bigcup_{i=1}^{m} g_{i} X=$ $\bigcup_{i=1}^{m} g_{i} X_{1} \cup \bigcup_{i=1}^{m} g_{i} X_{2}$. By Theorem 3.7, we may assume that $\bigcup_{i=1}^{m} g_{i} X_{1}$ is generic, so clearly $X_{1}$ is generic.

REMARK. Note that the converse to 3.8 is false. If $G=\left\langle\mathbb{R}^{2},+\right\rangle$ then $\left\{(x, y) \in \mathbb{R}^{2}:|y|>1\right\}$ is a generic set whose complement is not even bounded.

Also, Corollary 3.9 fails without the assumption on $G$. Indeed, take $G=$ $\langle\mathbb{R},+\rangle$. Then $G$ itself is the union of the negative and nonnegative elements, none of which is generic in $G$.

COROLlary 3.10. If $G$ is definably compact and commutative then there is a complete generic type $p(x) \in S(M)$ of $G$. That is, there is a type $p(x)$ in $G$ such that every formula in $p$ defines a generic subset of $G$.

\subsection{Stabilizers}

Definition 3.11. Let $X$ be a definable subset of $G$. By $\operatorname{Stab}(X)$ we mean $\{g \in G: X \triangle(g X)$ is nongeneric $\}$.

Lemma 3.12. If $G$ is definably compact and commutative then $\operatorname{Stab}(X)$ is a type-definable subgroup of $G$. $\in G$

Proof. First, $g \in \operatorname{Stab}(X)$ if and only if for each $m<\omega$, for all $g_{1}, \ldots, g_{m}$

$$
\bigcup_{i=1}^{m} g_{i}(X \triangle(g X)) \neq G .
$$

Hence $\operatorname{Stab}(X)$ is type-definable. Clearly $\operatorname{Stab}(X)$ is closed under inversion. By Corollary 3.9 it is also closed under multiplication, hence is a group.

Proposition 3.13. If $G$ is commutative then for every $n$ there is a definable set $X \subseteq G$ such that $\operatorname{Stab}(X)$ does not contain any point of order $n$.

Proof. Recall that we are assuming $G$ to be definably connected. It is well known that $G$ has only finitely many elements of order $n$ for all $n<\omega$. Hence $G$ is also divisible. Fix $n$. Let $f: G \rightarrow G$ be $f(x)=n x$. So $f$ is finite-to-one, say $r$-to-one, from $G$ onto $G$. So we can find a definable function $h: G \rightarrow G$ such that for any $a \in G, f(h(a))=a$. Let $X$ be the image of $h$. Thus for every $a \in G, X$ contains a unique $b$ such that $n b=a$. Let $c_{1}, \ldots, c_{r}$ be the points in $G$ of order $n$ (where $c_{1}=0$, say). Then we see that the sets $X, X+c_{2}, \ldots, X+c_{r}$ partition $G$. Let $H=\operatorname{Stab}(X)$. Then clearly $c_{i} \notin H$ for $i=2, \ldots, r$.

It follows that to prove that for $G$ commutative, $G^{00}$ is torsion-free, it will suffice to prove that for any definable set $X, \operatorname{Stab}(X)$ has bounded index in $G$. However, we were not able to prove that so far. We end this section 
with a conjecture, which is a strong version of Lemma 3.5 and if true, will imply the required result.

Conjecture A. Assume that $G$ is a definably compact commutative group defined over some model $\mathcal{M}_{0}$. If $X \subseteq G$ is generic in $G$ (but not necessarily definable over $\left.\mathcal{M}_{0}\right)$ then $X \cap G\left(\mathcal{M}_{0}\right)$ is nonempty.

The above conjecture, if true, taken together with the observations in (the proof of) Lemma 3.5 will imply the following analogue to a known result in the theory of stable groups:

If $G$ is a definably compact commutative group, defined over a model $\mathcal{M}_{0}$, and $X \subseteq G$ is definable then $X$ is generic in $G$ if and only if every translate of $X$ does not fork over $\mathcal{M}_{0}$.

Lemma 3.14. Assume that Conjecture $A$ is true. Suppose $G$ is commutative and definably compact. Then for every definable set $X \subseteq G$, the group $\operatorname{Stab}(X)$ has bounded index in $G$.

Proof. Suppose $G$ and $X$ to be definable over $\mathcal{M}_{0}$. Assume that $g, h \in G$ realize the same type over $\mathcal{M}_{0}$. Then, clearly, $X g \cap G\left(\mathcal{M}_{0}\right)=X h \cap G\left(\mathcal{M}_{0}\right)$, and hence $(X g \triangle X h) \cap G\left(\mathcal{M}_{0}\right)=\emptyset$. But, assuming Conjecture A, this implies that $X g \triangle X h$ is not generic in $G$ and hence $g h^{-1} \in \operatorname{Stab}(X)$.

Thus the number of cosets of $\operatorname{Stab}(X)$ in $G$ is at most $\left|S\left(\mathcal{M}_{0}\right)\right|$.

Recall that $G^{00}$ is the minimal type-definable subgroup of bounded index in $G$. By [2], this group indeed exists, it is divisible and the group $G / G^{00}$ is then a Lie group.

Lemma 3.15. Assume Conjecture $A$ to be true. Let $G$ be a definably compact commutative group. Then $G / G^{00}$ is a Lie group whose dimension equals $\operatorname{dim}(G)$.

Proof. By Proposition 3.13 and Lemma 3.14, the group $G^{00}$ is torsionfree. Since $G^{00}$ is divisible (see [2]) the number of $n$-torsion points of $G / G^{00}$, for every $n$, equals that of $G$. By Edmundo's theorem (see [6]) if $k=\operatorname{dim}(G)$, then the number of $n$-torsion points of $G$ equals the number of such points in $\left(S^{1}\right)^{k}$. Thus, $G / G^{00}$ must equal $\left(S^{1}\right)^{k}$.

\section{Nonstandard compact Lie groups, Haar measure, and gener-} ics. In this section we study generic sets in the special case where $G$ already "comes from" a compact Lie group. More precisely, we let $\mathcal{M}_{0}=$ $\langle\mathbb{R},\langle,+, \cdot, \cdots\rangle$ be an o-minimal expansion of the real field, and $H$ a connected compact Lie group definable in the structure $\mathcal{M}_{0}$. By the discussion in 1.1 and definable compactness we may assume $H$ to be a definable closed subset of $[-1,1]^{m}$ for some $m$ such that the group operation is continuous when $H$ is equipped with the induced topology. Assume that $\operatorname{dim}(H)=n$. 
By [5] there is a covering of $H$ by finitely many open definable subsets $U_{1}, \ldots, U_{k}$ and there are definable homeomorphisms $\phi_{i}: U_{i} \rightarrow V_{i}$ where each $V_{i}$ is an open definable subset of $[-r, r]^{n}$ (for some fixed $r \in \mathbb{R}$ ), the "transition maps" between the relevant open definable subsets of the $V_{i}$ are differentiable, and the group operation is differentiable when read in the $V_{i}$.

Now let $\mathcal{M}$ be a saturated o-minimal expansion of an elementary extension $\mathcal{M}_{1}$ of $\mathcal{M}_{0}$, and let $(G, \cdot)$ be the interpretation in $\mathcal{M}$ of the formulas defining the group $H$ in $\mathcal{M}_{0}$. Then $G$ is a definably compact group definable in $\mathcal{M}$. Let us write $H$ as $G(\mathbb{R})$. We have at our disposal the standard part map st : $G \rightarrow G(\mathbb{R})$. This is a surjective homomorphism whose kernel $\mu(e)$ is the infinitesimal neighbourhood of the identity $e \in G$. Namely $\mu(e)=\{x \in G: d(x, e)<1 / n$ for all $n \in \mathbb{N}\}$. We want to relate genericity of $\mathcal{M}$-definable subsets $X$ of $G$ to genericity of st $(X)$ in $G(\mathbb{R})$. We will use the existence of a Haar-like measure for definable subsets of $G$, which is maybe of interest in its own right. This was accomplished in the special case where $G=([0,1),+(\bmod 1))^{n}$ by Berarducci and Otero in [1], and their results almost immediately yield our generalization.

Before doing this it is worth recording that the conjectures of the second author from [9] hold for $G$ in the expected fashion. The proof when $G$ is definably simple (Proposition 3.6 of [9]) can be modified appropriately, but we give a brief proof. By a "torus" we mean here a connected compact commutative Lie group. (It is a basic fact about a compact Lie group $H$ that if $T$ is a maximal torus in $H$, i.e. $T$ is a closed subgroup of $H$ which is a torus and maximal such, then $T$ is a maximal abelian subgroup of $H$ and moreover the conjugates of $T$ cover $H$.)

FACT 4.1. Let $G$ be as above. Then:

(i) $G / \mu(e)$ equipped with the logic topology is homeomorphic (as a topological group) to the compact Lie group $G(\mathbb{R})$.

(ii) The o-minimal dimension of $G$ equals the Lie group dimension of $G(\mathbb{R})$.

(iii) $\mu(e)$ is the smallest type-definable subgroup of $G$ of bounded index (so equals $G^{00}$ ).

Proof. We only need to prove (iii). Let $T$ be a maximal torus of $G(\mathbb{R})$. Then $T$ is a maximal abelian subgroup of $G(\mathbb{R})$ so is definable in $\mathcal{M}_{0}$ as $Z(C(T))$ (using the DCC on definable subgroups). Let $T(\mathcal{M})$ be the interpretation of $T$ in $\mathcal{M}$. As $G(\mathbb{R})$ is covered by the conjugates of $T$, and $\mathcal{M}_{1}$ is an elementary extension of $\mathcal{M}_{0}, G$ is covered by the conjugates (by elements of $G$ ) of $T(\mathcal{M})$. 
Now both $G^{00}$ and $\mu(e)$ are normal subgroups of $G$ and $G^{00} \subseteq \mu(e)$. As $\mu(e) \cap T(\mathcal{M})$ is torsion-free, by Corollary 1.2 of [2] we have

$(*) \quad \mu(e) \cap T(\mathcal{M})=T(\mathcal{M})^{00}$ and thus $\quad \mu(e) \cap T(\mathcal{M})=G^{00} \cap T(\mathcal{M})$.

Now if $x \in \mu(e)$, then $x \in T(\mathcal{M})^{g}$ for some $g \in G$, so as $\mu(e)$ is normal in $G$, $x \in(\mu(e) \cap T(\mathcal{M}))^{g}$. By $(*)$ and the normality of $G^{00}, x \in G^{00}$. We have shown that $\mu(e)=G^{00}$.

We now recall the Haar measure. As $G(\mathbb{R})$ is a compact group it has a (both left and right) invariant countably additive measure $m$, normalized so that $m(G(\mathbb{R}))=1$. For compact Lie groups (such as $G(\mathbb{R})$ ) this Haar measure is given by an invariant differential $n$-form $\omega$ where $n$ is the dimension of the group. (See for example Section VII, Chapter V of [3].)

Now for $X$ a definable subset of $G$, define $h(X)=m(\operatorname{st}(X))$. Note that $\operatorname{st}(X)$ is closed so $h(X)$ is defined.

Proposition 4.2. $h$ is a finitely additive real-valued invariant measure on the Boolean algebra of definable subsets of $G$.

In order to prove the proposition, it suffices, as pointed out in [1], to show that if $X, Y$ are disjoint definable subsets of $G$ then $m(\operatorname{st}(X) \cap \operatorname{st}(Y))=0$. We proceed to prove this now. We need a couple of facts. Our notation $\left(H, U_{i}, \phi_{i}, V_{i}, \ldots\right)$ is as given above, and $|\cdot|$ stands for Lebesgue measure. First:

FACT 4.3. Let $X \subset H$ be closed and assume that $\left|\phi_{i}\left(X \cap U_{i}\right)\right|=0$ for each $i=1, \ldots, k$. Then $m(X)=0$.

Proof. By [3] again, $m\left(X \cap U_{i}\right)$ is given by integrating a suitable continuous function on $\mathbb{R}^{n}$ over $\phi_{i}\left(X \cap U_{i}\right)$. As the latter has Lebesgue measure 0 , $m\left(X \cap U_{i}\right)=0$, hence $m(X)=0$.

Secondly, we require the following results from [1] (Theorem 4.3 and Corollary 4.4 there):

FACT 4.4. Let $X, Y \subset \mathcal{M}^{n}$ be definable sets in $\mathcal{M}$. Assume that both $X$ and $Y$ are contained in the closed box $[-r, r]^{n}$ for some $r \in \mathbb{R}$. Then:

(i) $|\operatorname{st}(X)|>0$ if and only if $X$ contains a rectangular box whose vertices have rational coordinates.

(ii) If $X \cap Y=\emptyset$ then $|\operatorname{st}(X) \cap \operatorname{st}(Y)|=0$.

We now conclude:

Lemma 4.5. Let $X, Y$ be definable (in $\mathcal{M})$ subsets of $G$ with $X \cap Y=\emptyset$. Then $m(\operatorname{st}(X) \cap \operatorname{st}(Y))=0$. 
Proof. Assume that $m(\operatorname{st}(X) \cap \operatorname{st}(Y))>0$. Then by Fact 4.3 there is $i$ such that

$$
\left|\phi_{i}\left(\operatorname{st}(X) \cap \operatorname{st}(Y) \cap U_{i}\right)\right|>0 .
$$

Note that as $\phi_{i}$ is defined over $\mathbb{R}, \phi_{i}$ (when interpreted in $\mathcal{M}$ ) commutes with st $(-)$. So it follows from $(*)$ that $\left|\operatorname{st}\left(\phi_{i}\left(X \cap U_{i}(\mathcal{M})\right)\right) \cap \operatorname{st}\left(\phi_{i}\left(Y \cap U_{i}(\mathcal{M})\right)\right)\right|>0$. It follows from Fact 4.4(ii) that $\phi_{i}\left(X \cap U_{i}(\mathcal{M})\right) \cap \phi_{i}\left(Y \cap U_{i}(\mathcal{M})\right)$ is nonempty, which implies that $X \cap Y \neq \emptyset$.

Lemma 4.5 yields Proposition 4.2.

Proposition 4.6. Let $X \subset G$ be definable (in $\mathcal{M})$. Then the following are equivalent:

(i) $h(X)>0$,

(ii) $\operatorname{st}(X)$ has nonempty interior in $G(\mathbb{R})$.

(iii) $X$ contains a nonempty open set which is definable over $\mathcal{M}_{0}$.

(iv) $X$ is (left and right) generic in $G$.

(v) $\operatorname{st}(X)$ is (left and right) generic in $G(\mathbb{R})$.

Proof. (i) $\Rightarrow($ ii). By definition $m(\operatorname{st}(X))>0$. By Facts 4.3 and 4.4(i), $\operatorname{st}(X)$ contains an open subset of $G(\mathbb{R})$.

(ii) $\Rightarrow$ (iii). By abuse of notation we also use $U_{i}, \phi_{i}, V_{i}$ to denote the interpretation of these symbols in $\mathcal{M}$ when appropriate. As st $(X)$ contains an open subset of $G(\mathbb{R}),\left|\operatorname{st}\left(\phi_{i}\left(X \cap U_{i}\right)\right)\right|>0$ for some $i$. By Fact 4.4(i), $\phi_{i}\left(X \cap U_{i}\right)$ contains a rectangular open box with rational vertices. As $\phi_{i}$ is $\mathcal{M}_{0}$-definable it follows that $X \cap U_{i}$ and hence $X$ itself contains an open $\mathcal{M}_{0}$-definable subset of $G$.

(iii) $\Rightarrow$ (iv). Suppose that $Y$ is an $\mathcal{M}_{0^{-}}$-definable open (in $G$ ) subset of $X$. Then $Y(\mathbb{R})$ is an open subset of $G(\mathbb{R})$ so by compactness of $G(\mathbb{R}), Y(\mathbb{R})$ is generic in $G(\mathbb{R})$. By transfer, $Y$ is generic in $G$, hence $X$ is generic in $G$.

(iv) $\Rightarrow(\mathrm{v})$. This is immediate as st is a group homomorphism.

$(\mathrm{v}) \Rightarrow(\mathrm{i})$. This follows by additivity of Haar measure.

Note that Proposition 4.6 implies among other things that Conjecture A from Section 3 is true in the above setting.

We complete this section with the following:

Questions.

(i) Suppose $G$ is a definably compact group in a saturated o-minimal structure and that the second author's conjecture is true for $G$. Does the list of equivalences in Proposition 4.6 hold for definable subsets $X$ of $G$ (when $G(\mathbb{R})$ is replaced by $G / G^{00}$ and the standard part map by the quotient map)? 
(ii) Under the same assumptions, here is a special case of (i): Suppose $X$ is a definable subset of $G$ which meets every translate of $G^{00}$. Is $X$ generic in $G$ ?

5. Semigroups in definably compact groups. We work here in an o-minimal expansion of a real closed field (it is again sufficient to assume that $G$ is an affine group, with respect to its topology).

THEOREM 5.1. Let $G$ be a definable group, $S \subseteq G$ a definable semigroup in $G$ (i.e., a definable subset of $G$ closed under multiplication). If $\mathrm{Cl}_{G}(S)$ is definably compact then $S$ is necessarily a subgroup of $G$.

In particular, if $G$ is definably compact then every definable semigroup in $G$ is a subgroup of $G$.

Proof. We first prove the theorem in the case that $G$ is commutative. We further assume that $S$ is closed in $G$ and that $G$ is embedded in $M^{n}$ as a topological group. Consider the family $\mathcal{F}=\{g S: g \in S\}$. We claim that $\bigcap_{g \in S} g S$ is nonempty.

Indeed, $\mathcal{F}$ is a definable family of nonempty closed and bounded subsets of $M^{n}$, which is also directed. Namely, for every $g, h \in S$, the set $(g h) S$ is contained in both $g S$ and $h S$. Therefore, $\mathcal{F}$ has the finite intersection property and we may apply to it Corollary 2.2. Thus, there are finitely many elements of $G$ such that each $g S, g \in S$, contains one of those. But, since $\mathcal{F}$ is directed, one of these elements must be contained in all $g S, g \in S$.

So, we assume that $g_{0} \in g S$ for all $g \in S$, or equivalently,

$$
\forall g \in S \exists h \in S \quad g_{0}=g h .
$$

In particular, if we take $g=g_{0}^{2}$, then we may conclude that $g_{0}^{-1} \in S$.

Now, given any $g \in S$, we may write $g=g_{0} h^{-1}$ for some $h \in S$, and hence $g^{-1}=h g_{0}^{-1}$ is also in $S$, proving that $S$ is a subgroup of $G$.

Assume now that $S$ is not closed in $G$. Then, by continuity, $\mathrm{Cl}_{G}(S)$ is also a semigroup, which, by the above, must be a group. But now, $S$ is a large subset of the group $\mathrm{Cl}_{G}(S)$, and hence (see [8]), we must have $S \cdot S=\mathrm{Cl}_{G}(S)$. Since $S$ is a semigroup, it equals $\mathrm{Cl}_{G}(S)$, hence it is a group.

Consider now an arbitrary definable group $G$ (not necessarily commutative) and assume that $S$ is a definable semigroup in $G$ such that $\mathrm{Cl}_{G}(S)$ is definably compact. We will show that every element of $S$ has its inverse in $S$. Indeed, if $g \in S$ has finite order then the group generated by $g$ is contained in $S$. If $g$ has infinite order then consider the definable commutative group $H=Z\left(C_{G}(g)\right)$. Its intersection with $S$ is a nontrivial semigroup and hence by the above, $S$ contains $H$. In particular, $g^{-1} \in S$.

Remark. Clearly, if $G$ is not definably compact then it may have many definable semigroups. See, for example, the ray of positive elements in $\langle\mathbb{R},+\rangle$, or the semigroup of $\left\langle\mathbb{R}^{2},+\right\rangle$ defined by $\left\{(x, y) \in \mathbb{R}^{2}: x \geq 0 \&|y|<x^{2}\right\}$. 
We now obtain the following curious corollaries.

Corollary 5.2. Let $G$ be a definable group, $X \subseteq G$ a nonempty definable set such that $\mathrm{Cl}_{G}(X)$ is definably compact.

(i) If $g \in G$ and $g X \subseteq X$ then necessarily $g X=X$.

(ii) If $g \in G$ and $\operatorname{dim}(g X \backslash X) \leq r$ for some $r$, then $\operatorname{dim}(g X \triangle X) \leq r$.

Proof. (i) Let $S=\{h \in G: h X \subseteq X\}$. Then $S$ is a definable semigroup in $G$, containing $g$. Moreover, if $x \in X$ then $S x \subseteq X$ and therefore $\mathrm{Cl}_{G}(S)$ is definably compact. By Theorem $5.1, S$ is a subgroup of $G$, which easily implies that $h X=X$ for every $h \in S$.

(ii) Similarly, let $S=\{h \in G: \operatorname{dim}(h X \backslash X) \leq r\}$. It is easy to see that $S$ is a semigroup and therefore a group. Now, given $h \in S$, if $\operatorname{dim}(h X \backslash X) \leq r$ then also $\operatorname{dim}\left(h^{-1} X \backslash X\right) \leq r$, which implies that $\operatorname{dim}(X \triangle h X) \leq r$.

Corollary 5.3. Let $G$ be definably compact and commutative (written additively). Let $X \subseteq G$ be a definable 2-generic set and assume that for some $a \in G$ we have $\operatorname{dim}((a+X) \cap X)<\operatorname{dim}(X)$. Then:

(i) There is a torsion element $h \in G$ such that $\operatorname{dim}(G \backslash(X \cup(h+X))<$ $\operatorname{dim}(G)$.

(ii) Every translate of $X$ contains an element from $\mathcal{M}_{0}$.

Proof. Let $n=\operatorname{dim}(G)=\operatorname{dim}(X)$ and assume that $X \cup b+X=G$. By our assumptions, $\operatorname{dim}((a+X) \backslash(b+X))<n=\operatorname{dim}(G)$, and hence $\operatorname{dim}((a-b+X) \backslash X)<n$. By Corollary 5.2, $\operatorname{dim}((a-b+X) \triangle X)<n$. Let

$$
H=\{h \in G: \operatorname{dim}(h+X \triangle X)<n\} .
$$

Clearly $H$ is a definable subgroup of $G$.

Now, since $\operatorname{dim}(a+X \triangle b+X)<n$ and $\operatorname{dim}(X \cap a+X)<n$ we also have $\operatorname{dim}(b+X \cap X)<n$ and therefore $\operatorname{dim}(2 b+X \cap b+X)<n$. But $b+X \supseteq X^{\mathrm{c}}$, hence $\operatorname{dim}\left(2 b+X \cap X^{\mathrm{c}}\right)<n$, or equivalently $\operatorname{dim}(2 b+X \backslash X)<n$. It follows from 5.2 that $2 b \in H$ and therefore there is a natural number $k>1$ such that $k b \in H^{0}$ (where $H^{0}$ is the definably connected component of $H$ ).

Since $H^{0}$ is divisible, there is $h \in H^{0}$ such that $g=b-h$ is a torsion element of $G$ and we have $\operatorname{dim}(h+X \triangle X)<n$. It follows that $\operatorname{dim}(G \backslash$ $(X \cup g+X))<n$.

(ii) Let $Y=X \cup h+X$. By dimension considerations, every translate of $Y$ contains an element from $\mathcal{M}_{0}$. In particular $Y$ itself contains an element from $\mathcal{M}_{0}$. But if $h+X$ contains such an element then so does $X$ (since $\left.h \in \mathcal{M}_{0}\right)$.

6. Appendix: A proof of Theorem 2.1. For the sake of completeness, we present here a full proof of Theorem 2.1, extracted from Dolich's paper [4]. (6.1 and 6.3 are just Lemmas 4.5 and 4.6 from that paper, with 
somewhat extended proofs. Our 6.4 is a variation on Corollary 4.7 from his paper, and our Theorem 6.5 provides a direct proof of the theorem we need, based on the ideas from [4].)

Even though Dolich states his theorems under the assumption that the theory is "nice" (e.g. expands a real closed field), it is sufficient to assume for our purpose that $\mathcal{M}$ is an o-minimal structure with definable choice functions (e.g. an expansion of an ordered group).

Suppose $p(\bar{x})$ is a complete $n$-type (of dimension $n$ ) over $A$ and $q(y)$ is a complete 1-type over $A$. We will let $P, Q$ denote the sets of realizations of $p, q$ respectively. Note that if $\bar{a} \in P$, and $b \in Q$ and $b \in \operatorname{dcl}(a A)$, then this is witnessed by an $A$-definable function $f$ such that $f(\bar{a})=b$, and that $f$ is continuous and monotone (or constant) in each coordinate on some $A$-definable cell which contains $P$. We write $f: P \rightarrow Q$.

Lemma 6.1. Let $p(x), q(x)$ be complete nonalgebraic 1-types over a set $A$.

(a) Then either

(i) all $A$-definable $f: P \rightarrow Q$ are increasing (i.e. order-preserving: $x<y$ implies $f(x)<f(y))$, or

(ii) all $A$-definable $f: P \rightarrow Q$ are decreasing.

(b) In case (i) whenever $B \supseteq A, a \in P$ and $a>\operatorname{dcl}(B) \cap P$, then $\operatorname{dcl}(a A) \cap Q>\operatorname{dcl}(B) \cap Q$.

In case (ii), whenever $B \supseteq A, a \in P$ and $a<\operatorname{dcl}(B) \cap P$, then $\operatorname{dcl}(a A) \cap Q>\operatorname{dcl}(B) \cap Q$.

Proof. (a) Suppose for contradiction that there are $f: P \rightarrow Q$ and $g: P \rightarrow Q$ with $f$ increasing and $g$ decreasing. Let $h=g^{-1} f$. Then $h:$ $P \rightarrow P$ is decreasing (and is clearly a bijection). Now either $x<h(x)$ for all $x \in P$ or $x>h(x)$ for all $x \in P$. We suppose the first possibility and get a contradiction. (Likewise the second possibility will give a contradiction.) Fix $a \in P$. So $a<h(a)$. Let $c \in P$ be such that $h(c)=a$. So $h(c)<h(a)$ and as $h$ is decreasing, $a<c$. But then $c>h(c)$, contradicting our hypothesis.

(b) We assume case (i) of (a) holds. Suppose that $c \in \operatorname{dcl}(a A) \cap Q$, so this is witnessed by some $A$-definable $f: P \rightarrow Q$ with $f(a)=c$. Now $f$ is order-preserving and also gives a bijection between $\operatorname{dcl}(B) \cap P$ and $\operatorname{dcl}(B) \cap Q$. By choice of $a$ we see that $c>\operatorname{dcl}(B) \cap Q$.

In part (b) of the lemma we chose $a$ to realize one of two possible "canonical" extensions of $p$ to a complete type over $B$. We will generalize this notion to $n$-types.

Definition 6.2. Suppose $p\left(x_{1}, \ldots, x_{n}\right)$ is a complete $n$-type over $A$ of dimension $n$. Fix some sequence $\eta=(\eta(1), \ldots, \eta(n))$ where each $\eta(i)$ is 1 or 0 . Let $B \supseteq A$. We will define an extension $p_{B}^{\eta} \in S_{n}(B)$ of $p$. 
For $i=1, \ldots, n$ let $p_{i}\left(x_{1}, \ldots, x_{i}\right)$ be the restriction of $p$ to the variables $x_{1}, \ldots, x_{i}$. Let $\left(b_{1}, \ldots, b_{n}\right)$ be chosen as follows: $b_{1} \in P_{1}$ and if $\eta(1)=1$ then $b_{1}>\operatorname{dcl}(B) \cap P_{1}$, while if $\eta(1)=0$, then $b_{1}<\operatorname{dcl}(B) \cap P_{1}$.

Given $b_{1}, \ldots, b_{i}$ (which will realize $P_{i}$ ) let $b_{i+1} \in P_{i+1}\left(b_{1}, \ldots, b_{i}\right.$ ) (the set of realizations of $\left.p_{i+1}\left(b_{1}, \ldots, b_{i}, x_{i+1}\right)\right)$ be such that, if $\eta(i+1)=1$, then $b_{i+1}>\operatorname{dcl}\left(B, b_{1}, \ldots, b_{i}\right) \cap P_{i+1}\left(b_{1}, \ldots, b_{i}\right)$, and if $\eta(i+1)=0$, then $b_{i+1}<\operatorname{dcl}\left(B, b_{1}, \ldots, b_{i}\right) \cap P_{i+1}\left(b_{1}, \ldots, b_{i}\right)$.

Note (by o-minimality) that $\operatorname{tp}\left(b_{1}, \ldots, b_{n} / B\right)$ is an extension of $p$ which depends only on the choice of $\eta$. We define $p_{B}^{\eta}\left(x_{1}, \ldots, x_{n}\right)$ to be this type.

Proposition 6.3. Let $p\left(x_{1}, \ldots, x_{n}\right)$ be a complete $n$-type over $A$ of dimension n. Let $q(y)$ be a complete 1-type over $A$. Then there is $\eta$ as in Definition 6.2 such that for any $B \supseteq A$ and any realization $\bar{a}$ of $p_{B}^{\eta}$, $\operatorname{dcl}(\bar{a} A) \cap Q>\operatorname{dcl}(B) \cap Q$.

Proof. For simplicity we assume $A=\emptyset$. For $i=1, \ldots, n$, let $p_{i}\left(x_{1}, \ldots, x_{i}\right)$ be the restriction of $p$ to $x_{1}, \ldots, x_{i}$. Let $\left(a_{1}, \ldots, a_{n}\right)$ realize $p$. Let $P_{i}$ be the set of realizations of $p_{i}$, and $P_{i+1}\left(a_{1}, \ldots, a_{i}\right)$ be the set of realizations of $p_{i}\left(a_{1}, \ldots, a_{i}, x_{i+1}\right)$. We let $\bar{a}^{i}$ denote $\left(a_{1}, \ldots, a_{i}\right)$. So if $i=0$, then $\bar{a}^{i}=\emptyset$.

We first define $\eta$ and then show it works. Fix $1 \leq i+1 \leq n$ and we want to define $\eta(i+1)$. Let $q_{\bar{a}^{i}}(y)$ be the complete extension of $q$ over $\bar{a}^{i}$, determined by " $y<\operatorname{dcl}\left(\bar{a}^{i}\right) \cap Q$ ". (So if $i=0$, we just get the complete type $q$.) Let $Q\left(\bar{a}^{i}\right)$ be the set of realizations of $q_{\bar{a}^{i}}(y)$. Now if case (i) of Lemma 6.1(a) holds for $P_{i+1}\left(\bar{a}^{i}\right)$ and $Q\left(\bar{a}^{i}\right)$ (namely if all $\bar{a}^{i}$-definable functions between these two types are increasing) then define $\eta(i+1)=1$. Note that this does not depend on the choice of $\bar{a}^{i}$, only the fact that it realizes $p_{i}$. Otherwise, put $\eta(i+1)=0$. (Note it is possible that there are no such functions, increasing or decreasing, in which case any value for $\eta(i+1)$ will work.)

Now let $\left(b_{1}, \ldots, b_{n}\right)$ realize $p_{B}^{\eta}$. We prove by induction on $i=1, \ldots, n$ that

$$
\operatorname{dcl}\left(b_{1}, \ldots, b_{i}\right) \cap Q>\operatorname{dcl}(B) \cap Q .
$$

For $i=1$, this is given by Lemma 6.1(b). Now for the inductive step. Our inductive hypothesis is $(*)$. Let $\bar{b}^{i}$ denote $\left(b_{1}, \ldots, b_{i}\right)$. Suppose that $c \in \operatorname{dcl}\left(\bar{b}^{i}, b_{i+1}\right)$. If $c>d$ for some $d \in \operatorname{dcl}\left(\bar{b}^{i}\right) \cap Q$ then by the inductive hypothesis $(*), c>\operatorname{dcl}(B) \cap Q$. Otherwise, clearly $c$ realizes $Q\left(\bar{b}^{i}\right)$. But then $b_{i+1} \in P_{i+1}\left(\bar{b}^{i}\right)$, and $b_{i+1}>\operatorname{dcl}\left(B \bar{b}^{i}\right) \cap P_{i+1}\left(\bar{b}^{i}\right)$ if $\eta(i+1)=0$, and $b_{i+1}<\operatorname{dcl}\left(B \bar{b}^{i}\right) \cap P_{i+1}\left(\bar{b}^{i}\right)$ if $\eta(i+1)=1$. So by Lemma $6.1(\mathrm{~b})$, and the way we chose $\eta(i+1)$, it follows that $c>\operatorname{dcl}\left(B \bar{b}^{i}\right) \cap Q\left(\bar{b}^{i}\right)$. But then, by the definition of $Q\left(\bar{b}^{i}\right)$ and the inductive hypothesis $(*)$, we see that $c>\operatorname{dcl}(B) \cap Q$.

EXAMPLES. We work in the structure $M=\langle\mathbb{R},+, \cdot\rangle$.

(1) Let

$$
P(x, y)=\{x>n: n \in \mathbb{N}\} \cup\{y>f(x): f \text { is 0-definable }\}
$$


and $Q(x)=\{x>n: n \in \mathbb{N}\}$. Then the associated $\eta$ can be either $(1,0)$ or $(1,1)$.

(2) Let

$$
\begin{aligned}
P(x, y)= & \{x>n: n \in \mathbb{N}\} \cup\{y>n: n \in \mathbb{N}\} \\
& \cup\left\{y<f(x): f \text { is 0-definable and } \lim _{t \rightarrow+\infty} f(t)=+\infty\right\}
\end{aligned}
$$

and $Q(x)$ as above. Then the associated $\eta$ must be $(1,1)$.

The next step is the following version of Corollary 4.7 from Dolich's paper.

LEMMA 6.4. Let $X(a)$ be a closed and bounded subset of $M^{n+1}$, defined over an independent tuple $a \in M^{m}$, and assume that $X(a) \cap \operatorname{dcl}(\emptyset)=\emptyset$. Let $p=\operatorname{tp}(a / \emptyset)$, and for $\eta \in 2^{m}$, let $a_{\eta} \models p_{a}^{\eta}$. Let $\pi: M^{n+1} \rightarrow M^{n}$ be the projection on the first $n$ coordinates. Then the set

$$
\pi\left(X(a) \cap \bigcap_{\eta \in 2^{m}} X\left(a_{\eta}\right)\right)
$$

does not contain any element of $\operatorname{dcl}(\emptyset) \cap M^{n}$.

Proof. Fix $c \in \operatorname{dcl}(\emptyset) \cap M^{n}$ and consider the set $I(a)=\left\{x \in M: c^{x} \in\right.$ $X(a)\}$. It is a finite union of closed intervals, $I_{1}(a)<\cdots<I_{k}(a)$, none containing a point in $\operatorname{dcl}(\emptyset)$. Thus each $I_{j}$ is contained in the realization set of a complete nonalgebraic type $q_{j} \in S_{1}(\emptyset)$.

For each $j=1, \ldots, k$, choose $\eta_{j}$ as given by Proposition 6.3 , for the types $P$ and $Q_{j}$. For simplicity, write $a_{j}=a_{\eta_{j}}$.

The endpoints of the interval $I_{j}(a)$ are in $\operatorname{dcl}(a) \cap Q_{j}$, while the endpoints of $I_{j}\left(a_{j}\right)$ are in $\operatorname{dcl}\left(a_{j}\right) \cap Q_{j}$. Hence, by Lemma 6.1 (with $a$ for $B$ ), the endpoints of $I_{j}\left(a_{j}\right)$ lie above the endpoints of $I_{j}(a)$. Moreover, if $q_{i}=q_{j}$ then the endpoints of $I_{j}\left(a_{j}\right)$ lie above the endpoints of $I_{i}(a)$ as well. In particular, $I_{j}\left(a_{j}\right) \cap I(a) \cap Q_{j}=\emptyset$. Clearly, if $q_{i} \neq q_{j}$ then $I_{j}\left(a_{j}\right) \cap I(a)=\emptyset$, and thus $I\left(a_{j}\right) \cap I(a) \subseteq \bigcup_{i \neq j} Q_{i}$.

Doing that for every $j$, one has $I(a) \cap \bigcap_{\eta \in 2^{m}} I\left(a_{\eta}\right)=\emptyset$ and thus $c \notin$ $\pi\left(X(a) \cap \bigcap_{\eta \in 2^{m}} X\left(a_{\eta}\right)\right)$.

Theorem 6.5. Assume that $\{X(a): a \in S\}$ is an $\mathcal{M}_{0}$-definable family of closed and bounded subsets of $M^{n}$. Let $p(x) \in S_{m}\left(\mathcal{M}_{0}\right)$ be a type of some $a \in S$, let $P=p(M)$. Then $\{X(a): a \in P\}$ has the finite intersection property if and only if there is $c \in \mathcal{M}_{0}$ such that $c \in X(a)$ for every $a \in P$.

Proof. The "if" direction of the theorem is obvious.

Consider $\{X(a): a \in P\}$, a family of closed and bounded subsets of $M^{n}$ with the finite intersection property. Clearly, one may assume that $p$ is an open type (by just considering the independent coordinates in $p$ ). By extending the language we may assume that $\mathcal{M}_{0}=\operatorname{dcl}(\emptyset)$. 
The proof is done by induction on $n$. The case $n=1$ will be reduced in this way to the case $n=0$, in which $X(a)$ clearly contains a single point.

Fix $a \in P$ and assume towards a contradiction that $X(a) \cap \operatorname{dcl}(\emptyset)=\emptyset$.

For $\eta_{i} \in 2^{m}$ let $a_{i} \models p_{a}^{\eta_{i}}$. Consider the type $\bar{P}=\operatorname{tp}\left(a, a_{1}, \ldots, a_{2^{m}} / \emptyset\right)$, and the family

$$
\left\{\pi\left(X(b) \cap \bigcap_{i=1}^{2^{m}} X\left(b_{i}\right)\right):\left(b, b_{1}, \ldots, b_{2^{m}}\right) \models \bar{P}\right\} .
$$

This is a family of closed and bounded subsets of $M^{n-1}$, with the finite intersection property (since so was our original family). By induction each such set contains an element of $\operatorname{dcl}(\emptyset)$.

However, by Lemma 6.4, the set $\pi\left(X(a) \cap \bigcap_{\eta \in 2^{m}} X\left(a_{\eta}\right)\right) \cap \operatorname{dcl}(\emptyset)$ is empty, a contradiction.

REMARK. Notice that the proof of Dolich's theorem gives an upper bound on the needed $N$ such that $N$-consistency of $\{X(a): a \in P\}$ implies the existence of a point in $\mathcal{M}_{0} \cap X(a)$.

Namely, for every $m, n \in \mathbb{N}$, there is $N=N(m, n)$ such that for every complete type $p$ over $\mathcal{M}_{0}$ of dimension $m$ and for every definable family $\{X(a): a \in P\}$ of closed and bounded subsets of $M^{n}$, if the family is $N$-consistent then it is finitely consistent.

Indeed, as the proof shows, if $X(a)$ does not contain a point in $\mathcal{M}_{0}$ then one can produce a family of closed and bounded subsets of $M^{n-1}$ using the projection of $1+2^{m}$ sets from the original family, such that none of the sets contains a point in $\mathcal{M}_{0}$. This new family is parameterized by a type of dimension at most $2^{m}$. Proceeding in this way, we may get $N=$ $\left(1+2^{m}\right) \cdot\left(1+2^{2^{m}}\right) \cdots$, where the product has length $n$.

\section{References}

[1] A. Berarducci and M. Otero, An additive measure in o-minimal expansions of fields Quart. J. Math. 55 (2004), 411-419.

[2] A. Berarducci, M. Otero, Y. Peterzil and A. Pillay, A descending chain condition for groups definable in o-minimal structures, Ann. Pure Appl. Logic 134 (2005), 303-313.

[3] C. Chevalley, Theory of Lie Groups I, Princeton Univ. Press, 1946.

[4] A. Dolich, Forking and independence in o-minimal theories, J. Symbolic Logic 69 (2004), 215-240.

[5] L. van den Dries, Tame Topology and o-Minimal Structures, London Math. Soc. Lecture Notes Ser. 248, Cambridge Univ. Press, 1998.

[6] M. Edmundo, o-Minimal cohomology and definably compact definable groups, preprint.

[7] Y. Peterzil and C. Steinhorn, Definable compactness and definable subgroups of ominimal groups, J. London Math. Soc. 59 (1999), 769-786. 
[8] A. Pillay, On groups and fields definable in o-minimal structures, J. Pure Appl. Algebra 53 (1988), 239-255.

[9] - Type-definability, compact Lie groups, and o-minimality, J. Math. Logic 4 (2004), 147-162.

Department of Mathematics

Department of Mathematics

University of Haifa

Haifa, Israel

UIUC

E-mail: kobi@math.haifa.ac.il

Urbana, IL 61801, U.S.A.

E-mail: pillay@math.uiuc.edu

and

School of Mathematics

University of Leeds

Leeds LS2 9JT, UK

E-mail: pillay@maths.leeds.ac.uk

Received 2 April 2006;

in revised form 2 October 2006 